\title{
Micro-costing analysis of guideline-based treatment by direct-acting agents: the real- life case of hepatitis $C$ management in Brazil
}

Hugo Perazzo ${ }^{1 *}$, Marcelino Jose Jorge ${ }^{2}$, Julio Castro Silva ${ }^{3}$, Alexandre Monken Avellar ${ }^{2}$, Patrícia Santos Silva², Carmen Romero ${ }^{4}$, Valdilea Gonçalves Veloso ${ }^{1}$, Ruben Mujica-Mota ${ }^{5}$, Rob Anderson ${ }^{5}$, Chris Hyde ${ }^{5}$ and Rodolfo Castro ${ }^{1,6}$

\begin{abstract}
Background: Eradication of hepatitis C virus (HCV) using direct-acting agents (DAA) has been associated with a financial burden to health authorities worldwide. We aimed to evaluate the guideline-based treatment costs by DAAs from the perspective of the Brazilian Ministry of Health (BMoH).

Methods: The activity based costing method was used to estimate the cost for monitoring/treatment of genotype-1 (GT1) HCV patients by the following strategies: peg-interferon (PEG-IFN)/ribavirin (RBV) for 48 weeks, PEG-IFN/RBV plus boceprevir (BOC) or telaprevir (TEL) for 48 weeks, and sofosbuvir (SOF) plus daclastavir (DCV) or simeprevir (SIM) for 12 weeks. Costs were reported in United States Dollars without (US\$) and with adjustment for purchasing power parity (PPP\$). Drug costs were collected at the National Database of Health Prices and an overview of the literature was performed to assess effectiveness of SOF/DCV and SOF/SIM regimens in real-world cohorts.
\end{abstract}

Results: Treatment costs of GT1-HCV patients were PPP\$ 43,176.28 (US\$24,020.16) for PEG-IFN/RBV, PPP\$ 71,196.03 (US\$ 39,578.23) for PEG-IFN/RBV/BOC and PPP\$ 86,250.33 (US\$ 47,946.92) for PEG-IFN/RBV/TEL. Treatment by all-oral interferon-free regimens were the less expensive approach: PPP\$ 19,761.72 (US\$10,985. 90) for SOF/DCV and PPP\$ 21,590.91 (US\$ 12,002.75) for SOF/SIM. The overview reported HCV eradication in up to $98 \%$ for SOF/DCV and $96 \%$ for SOF/SIM.

Conclusion: Strategies with all oral interferon-free might lead to lower costs for management of GT1-HCV patients compared to IFN-based regimens in Brazil. This occurred mainly because of high discounts over international DAA prices due to negotiation between $\mathrm{BMoH}$ and pharmaceutical industries.

Keywords: Chronic hepatitis C, Treatment, Micro-costing

\section{Background}

Chronic hepatitis $\mathrm{C}(\mathrm{CHC})$ remains a major public health issue that might lead to cirrhosis and its complications, such as portal hypertension and hepatocellular carcinoma [1]. Eradication of hepatitis $\mathrm{C}$ virus ( $\mathrm{HCV}$ ) has been associated with lower rates of liver-related complications

\footnotetext{
* Correspondence: perazzohugo@gmail.com; hugo.perazzo@ini.fiocruz.br ${ }^{1}$ Fundação Oswaldo Cruz, FIOCRUZ, Instituto Nacional de Infectologia Evandro Chagas, INI, Laboratório de Pesquisa Clínica em DST e AIDS, LAPCLIN-AIDS, Rio de Janeiro, Brazil

Full list of author information is available at the end of the article
}

[2]. Transient elastography (TE) by FibroScan ${ }^{\circledR}$ (EchoSens, Paris, France) is a validated method to liver fibrosis assessment [3] and seems to be cost-effective to diagnose cirrhosis compared to liver biopsy [4]. The high efficacy of direct-acting antiviral drugs (DAAs) has revolutionized the management of patients with $\mathrm{CHC}$. High rates of sustained virological response (SVR), defined as undetectable serum $\mathrm{HCV}$ RNA levels 12 weeks after completing treatment, has been obtained by DAA treatment in patients with advanced liver fibrosis or compensate cirrhosis [5]. 
However, the decrease of the burden of liver disease in $\mathrm{CHC}$ by DAA treatment might be associated with high costs to health authorities worldwide [6]. Public health strategies should be implemented to promote primary care access to interferon-free regimes, especially in low to middle-income countries with high prevalence of $\mathrm{CHC}$.

Drug prices are very important in $\mathrm{HCV}$ management but they are clearly not the only costs to be considered in the analysis [7]. The decision to incorporate new drugs in guidelines for $\mathrm{CHC}$ management should take into account the cost and cost-effectiveness analysis. The evidence base from economic evaluations of new healthcare technologies has been limited by the quality of the available data, strengths and weaknesses of different methods used for cost determination and differences among public health systems worldwide [8]. In Brazil, it is estimated that 1.4 to 1.7 million of people are living with $\mathrm{CHC}$ [9], most of them genotype-1 (GT1) HCV [10] and up to $20 \%$ with advanced fibrosis or cirrhosis [11]. Recently, the Brazilian Ministry of Health (BMoH) has recommended the use of DAAs for $\mathrm{CHC}$ treatment due to high discounts given by pharmaceutical companies. Since July 2015, patients with advanced fibrosis (METAVIR $\mathrm{F} \geq 3$ ) have free access to these drugs [12]. However, few studies have investigated the cost for $\mathrm{CHC}$ treatment with DAAs in a low to middle-income country. The aim of this study was to evaluate the guidelinebased treatment costs by DAAs from the perspective of a public national reference healthcare institute in Brazil. In addition, we reviewed the literature for effectiveness of all-oral interferon-free regimens available in Brazil in real-life cohort studies.

\section{Methods}

\section{Study design and setting}

This cost-analysis study used cost data collected in a tertiary center for treatment of infectious diseases: National Institute of Infectious Diseases Evandro Chagas-Oswaldo Cruz Foundation (INI/FIOCRUZ) at Rio de Janeiro, Brazil. INI/FIOCRUZ has been playing an important role for the $\mathrm{BMoH}$ in development of public health policies and strategies for prevention and treatment of infectious diseases, including viral hepatitis. In the Brazilian Public Health System, known as Sistema Único de Saúde (SUS), individuals can have free access to HCV treatment whether confirmed presence of liver fibrosis. According to the recommendations for $\mathrm{HCV}$ treatment from the $\mathrm{BMoH}$, treatment was delivery for free in patients with liver fibrosis stages $F \geq 2$ until 2012. However, from 2013 to nowadays, the threshold for free-treatment access has changed for fibrosis stages $F \geq 3$. This analysis simulated follow-up and antiviral regimens exclusively for treatment of GT1-
$\mathrm{HCV}$ patients according to the recommendation of $\mathrm{BMoH}$ from 2011 to 2015.

\section{Method for cost determination}

The activity based costing $(\mathrm{ABC})$ method was used for cost determination. This micro-costing method identifies different activities that the institution performs and includes the corresponding indirect costs (e.g. management costs) to estimate total cost of a procedure. ABC method recognizes the relationship between costs, activities (called cost drivers) and products (exams or medical visit) taking into account this relationship in the costing analysis [13]. Secondary data were obtained from: (i) public access production resources from the Department of Statistics; (ii) inventory of permanent material; (iii) records of expenditures with resources from the Financial Department for a one-year (2013) period (to correct the potential seasonal expenses due to diseases outbreaks in infectious diseases); and (iv) cost of 450 diagnostic tests and 100 sub-types of clinical procedures. Administrative costs were estimated through the determination of the proportional contribution of each activity to the pooled expenses and considered for all diagnostic tests and clinical procedures. Prices in national currency [Brazilian Real (BRL)] and inflated to average prices of July of 2015 in United States Dollars according to the World Bank, without (exchange rate $=3.11$ ) and with adjustment for purchasing power parity (PPP adjustment rate $=1.73$ ). We report these as the "nominal price" (US\$) and the "PPP-adjusted price" (PPP\$), respectively.

Costs of personnel, blood analysis tests and medical visits Personnel costs for each procedure were calculated based on the annual salary payment of each employee and the working time spent in the specific activity. At INI/FIOCRUZ, salaries are determined according to employee's specialization (i.e., people with $\mathrm{PhD}$ degree have higher salaries compared to those without) for individuals working in similar activities. Regarding expenditures with blood analysis tests, we considered costs of materials needed to perform the test, such as kits and reagents. Methodology for cost determination of non-invasive methods for liver fibrosis assessment were detailed in the Supplementary Material. Briefly, the following aspects were taken into account to estimate the cost per unit of TE (i) the price and depreciation of the FibroScan ${ }^{\circledR}$ machine; (ii) annual maintenance by $M$ probe calibration; (iii) salaries of personnel who perform the examination and (iv) indirect administrative costs. We reported the price of TE adjusted with the opportunity cost of using public resources for implementation of new technologies (cost of capital). Costs with specific material for liver biopsy [Menghini needle 16G (Biomedical, Florence, 
Italy)] and histological analysis, as well as personnel costs and cost for a day-clinic hospitalization were considered for estimation of total cost to perform a liver biopsy. The market prices were considered for diagnostic tests not performed at INI/FIOCRUZ.

\section{Cost of antiviral drugs for $\mathrm{CHC}$ treatment}

Drug costs were collected in a web-based public domain site that describes the prices paid by the $\mathrm{BMoH}$ for all drugs incorporated in the Public Health System and purchased from the pharmaceutical industry (Database of Health Prices "Banco de Preços em Saúde" - accessible at: http://www.saude.gov.br/bps). A weighted-mean price for each antiviral drug was estimated taking into account all drug payments made by the $\mathrm{BMoH}$ from January to December 2015. We then calculated the price for a single-dose of PEG-interferon $\alpha$-2a 180 mcg (PEG-IFN) [Pegasys ${ }^{\oplus}$ - Roche] and a single capsule or tablet for ribavirin (RBV) [generic by $\mathrm{BMoH}$ ], boceprevir (BOC) [Victrelis ${ }^{\oplus}$ - Merk], telaprevir (TEL) [Incivek ${ }^{\oplus}$ - Vertex Pharmaceuticals], sofosbuvir (SOF) [Sovaldi $^{\odot}$ - Gilead], daclastavir (DCV) [Daklinza ${ }^{\oplus}$ Bristol-Myers Squibb] and simeprevir (SIM) Olysio ${ }^{\bullet}-$ Janssen Pharmaceuticals].

\section{Strategies for treatment of GT1-HCV patients according to the $\mathrm{BMoH}$ guidelines}

In the present study, we compared the strategies for HCV treatment of GT1 patients as recommended by the $\mathrm{BMoH}$ from 2011 to 2015. During this period, Brazilian guidelines for $\mathrm{CHC}$ treatment were published in 2011, 2013 and 2015. In 2011, GT1 patients should be treated by PEG-IFN plus RBV for 48 weeks and a liver biopsy with significant fibrosis (METAVIR $\mathrm{F} \geq 2$ ) was necessary for treatment access (regimen $\mathrm{BMoH}-2011$ ) [14]. In 2013, presence of advanced fibrosis (liver biopsy METAVIR $F \geq 3$ or $\mathrm{TE} \geq 9.6 \mathrm{kPa}$ ) allowed the addition of first generation protease inhibitors [BOC for 44 weeks (regimen BMoH-2013A) or TEL for 12 weeks (regimen $\mathrm{BMoH}-2013 \mathrm{~B})]$ to PEG-IFN/RBV during 48 weeks [15]. More recently, in 2015, all-oral and interferon-free regimens [SOF plus DCV for 12 weeks (regimen BMoH-2015A) or SOF plus SIM for 12 weeks (regimen BMoH-2015B)] were recommended for GT1 $\mathrm{HCV}$ patients. Patients with decompensated cirrhosis, HIV co-infection or previous failure with PEG-IFN/ $\mathrm{RBV} \pm \mathrm{BOC}$ or TEL might be treated by SOF/DCV 24 weeks. In addition, serological biomarkers or TE could replace liver biopsy for free-access to DAA treatment [12]. In all scenarios, liver fibrosis was staged for treatment access and patients were followed with blood tests and medical visits. Table 1 summarizes the strategies for treatment of GT1 HCV patients according to the $\mathrm{BMoH}$ guidelines from 2011 to 2015 .

\section{Literature review for efficacy of SOF/DCV and SOF/SIM regimens in real-life scenario}

We systematically searched the references using the following predetermined inclusion criteria: cohort study that included GT1 HCV patients treated in real-life scenario by $\mathrm{SOF} / \mathrm{DCV}$ or $\mathrm{SOF} / \mathrm{SIM} \pm \mathrm{RBV}$ for 12 weeks. This search was performed in June 2017 at MEDLINE (PubMed) (1966-2017) using the following terms: ["sofosbuvir" OR "Sovaldi" OR "GS-7977" OR "simeprevir" OR "Olysio" OR"TMC-435" OR "daclastavir" OR "Daklinza" OR "BMS-790052"] AND ["hepatitis C"] AND ["realworld" OR "real-life"] (Additional file 1: Table S1).

\section{Results}

\section{Costs for liver fibrosis assessment}

The estimated costs of TE with personnel, machine and administrative costs were PPP\$ 40.08, 29.80 and 24.78, respectively. The nominal and PPP-adjusted costs of a single TE exam were PPP\$ 95.32 (US\$ 53.32). The adjustment using the opportunity cost lead to an increase in up 50\% of TE price (Additional file 1: Table S2). Costs of APRI and FIB-4 were estimated at PPP\$ 27.85 (US\$ 15.48) and PPP\$ 36.58 (US\$ 20.33), respectively. Total cost for liver biopsy was PPP\$ 834.41 (US\$ 482.32) including material to perform liver biopsy and histological analysis [PPP\$ 80.75 (US\$ 46.68)], personnel costs [PPP\$ 301.47 (US\$ 174.26)] and cost for day-clinic hospitalization [PPP\$ 452.19 (US\$ 261.38)].

\section{Costs for GT1 HCV treatment}

The estimated price of GT1 HCV treatment drugs was an important source of variability in the total cost for HCV treatment. PEG-IFN $\alpha-2 \mathrm{a} 180 \mathrm{mcg}$ cost PPP\$ 648.00 (US\$ 360.22) per weekly injection and RBV was the least expensive drug costing PPP\$ 106.05 (US\$ 58.80) per week. The regimen PEG-IFN (180 mcg SC weekly)/RBV (1250 mg PO daily if body weight $\geq 75 \mathrm{~kg}$ ) cost PPP\$ 754.05 or US\$ 419.18 per week. Considering the first generation protease inhibitors (PI), TEL had a higher cost compared to BOC [PPP\$ 87.01 (US\$ 48.37) vs PPP\$ 7.96 (US\$ 4.42) per tablet). The weekly costs for treatment by BOC (800 mg PO q8hr) and TEL (750 mg PO q8hr) were PPP\$ 668.64 and 3654.42, respectively. Thus, the weekly costs for PEG-IFN/first generation PI regimens ranges from PPP\$ 1422.69 (with BOC) and 4408.47 (with TEL). Considering the DAAs, the NS5A inhibitor DCV $60 \mathrm{mg}$ and the second generation PI SIM $150 \mathrm{mg}$ cost PPP\$ 388.78 (US\$ 206.09) and PPP\$ 541.24 (US\$ 300.86) per week, respectively. In addition, SOF $400 \mathrm{mg}$, a NS5B polymerase inhibitor, had a PPPadjusted and a nominal cost of PPP\$ 1014.23 and US\$ 563.78 weekly, respectively. The regimens SOF (400 mg PO daily)/DCV (60 mg PO daily) and SOF (400 mg PO daily)/SIM (150 mg PO daily) cost PPP\$ 1403.01 and 
Table 1 Description of strategies for treatment of genotype 1 chronic hepatitis C according to the Brazilian Ministry of Health from 2011 to 2015

\begin{tabular}{|c|c|c|c|c|c|c|}
\hline Guideline & $\begin{array}{l}\text { Fibrosis } \\
\text { staging }\end{array}$ & $\begin{array}{l}\text { Prerequisite } \\
\text { for treatment }\end{array}$ & Drugs & $\begin{array}{l}\text { Duration } \\
\text { (weeks) }\end{array}$ & Doses & Interventions \\
\hline \multirow[t]{2}{*}{$\begin{array}{l}\mathrm{BMoH}- \\
2011\end{array}$} & \multirow[t]{2}{*}{$\begin{array}{l}\text { Liver } \\
\text { biopsy }\end{array}$} & \multirow[t]{2}{*}{$\begin{array}{l}\text { METAVIR } \\
F \geq 2\end{array}$} & $\begin{array}{l}\text { PEG-IFN a-2a } 180 \text { mcg } \\
{\left[\text { Pegasys }^{\oplus} \text { - Roche }\right]}\end{array}$ & 48 & $\begin{array}{l}1 \\
\text { injection } \\
\text { weekly }\end{array}$ & \multirow{2}{*}{$\begin{array}{l}\text { PEG-RBV for } 48 \text { weeks. Medical visit at screening, baseline, W2, W4 } \\
\text { and every } 4 \text { weeks for W4 to W48. Response-guided therapy or } \\
\text { stopping rules based on HCV-RNA at W4, W12 and W24. SVR } \\
\text { at W72 }\end{array}$} \\
\hline & & & $\begin{array}{l}\text { RBV } 250 \text { mg } \\
\text { [BMoH generic] }\end{array}$ & 48 & $\begin{array}{l}5 \\
\text { capsules } \\
\text { daily }\end{array}$ & \\
\hline \multirow[t]{3}{*}{$\begin{array}{l}\text { BMoH- } \\
2013 \mathrm{~A}\end{array}$} & \multirow[t]{3}{*}{ TE } & \multirow[t]{3}{*}{$\begin{array}{l}\mathrm{LSM} \geq \\
9.6 \mathrm{kPa}\end{array}$} & $\begin{array}{l}\text { PEG-IFN a-2a } 180 \mathrm{mcg} \\
\text { [Pegasys }^{\oplus} \text { - Roche] }\end{array}$ & 48 & $\begin{array}{l}1 \\
\text { injection } \\
\text { weekly }\end{array}$ & \multirow{3}{*}{$\begin{array}{l}\text { Leading-phase with PEG-RBV for } 4 \text { weeks and PEG-RBV/BOC for } \\
44 \text { weeks. Medical visit at screening, baseline, W2, W4, W5, W6 } \\
\text { and W8, and every } 4 \text { weeks from W8 to W48. Response-guided } \\
\text { therapy or stopping rules based on HCV-RNA on W4, W12 and } \\
\text { W24. SVR at W72 }\end{array}$} \\
\hline & & & $\begin{array}{l}\text { RBV } 250 \text { mg } \\
\text { [BMoHgeneric] }\end{array}$ & 48 & $\begin{array}{l}5 \\
\text { capsules } \\
\text { daily }\end{array}$ & \\
\hline & & & $\begin{array}{l}\text { BOC } 200 \text { mg } \\
\text { Victrelis }^{\oplus}-\text { Merk] }\end{array}$ & 44 & $\begin{array}{l}12 \\
\text { tablets } \\
\text { daily }\end{array}$ & \\
\hline \multirow[t]{3}{*}{$\begin{array}{l}\text { BMoH- } \\
2013 \mathrm{~B}\end{array}$} & \multirow[t]{3}{*}{ TE } & \multirow[t]{3}{*}{$\begin{array}{l}\mathrm{LSM} \geq \\
9.6 \mathrm{kPa}\end{array}$} & $\begin{array}{l}\text { PEG-IFN a-2a } 180 \mathrm{mcg} \\
\text { [Pegasys }^{\oplus} \text { - Roche] }\end{array}$ & 48 & $\begin{array}{l}1 \\
\text { injection } \\
\text { weekly }\end{array}$ & \multirow{3}{*}{$\begin{array}{l}\text { PEG-RBV/TEL for } 12 \text { weeks and PEG-RBV for } 36 \text { weeks. Medical } \\
\text { visit at at screening, baseline, W1, W2 and W4, then every } 4 \\
\text { weeks from W4 to W48. Response-guided therapy or stopping } \\
\text { rules based on HCV-RNA on W4, W12 and W24. SVR at W72 }\end{array}$} \\
\hline & & & $\begin{array}{l}\text { RBV } 250 \text { mg } \\
\text { [BMoH generic] }\end{array}$ & 48 & $\begin{array}{l}5 \\
\text { capsules } \\
\text { daily }\end{array}$ & \\
\hline & & & $\begin{array}{l}\text { TEL } 375 \text { mg } \\
\text { [Incivek }{ }^{\circledR} \text { - Vertex] }\end{array}$ & 12 & $\begin{array}{l}6 \\
\text { tablets } \\
\text { daily }\end{array}$ & \\
\hline \multirow[t]{2}{*}{$\begin{array}{l}\text { BMoH- } \\
2015 \mathrm{~A}\end{array}$} & \multirow[t]{2}{*}{$\begin{array}{l}\text { TE or } \\
\text { APRI or } \\
\text { FIB-4 }\end{array}$} & \multirow{2}{*}{$\begin{array}{l}\mathrm{LSM} \geq 9.6 \\
\mathrm{kPa} \text { or } \mathrm{APRI} \\
\geq 1.5 \text { or } \\
\mathrm{FIB}-4 \geq 3.25\end{array}$} & $\begin{array}{l}\text { SOF } 400 \text { mg } \\
\text { [Sovaldi甲 - Gilead] }\end{array}$ & 12 & $\begin{array}{l}1 \\
\text { tablet } \\
\text { daily }\end{array}$ & \multirow[t]{2}{*}{$\begin{array}{l}\text { SOF/DCV for } 12 \text { weeks. Medical visits at screening, baseline, W2, } \\
\text { W4, W8 and W12. No response-guided therapy or stopping rules. } \\
\text { SVR at W24 }\end{array}$} \\
\hline & & & $\begin{array}{l}\text { DCV } 60 \text { mg } \\
\text { [Daklinza }^{\oplus} \text { - BMS] }\end{array}$ & 12 & $\begin{array}{l}1 \\
\text { tablet } \\
\text { daily }\end{array}$ & \\
\hline \multirow[t]{2}{*}{$\begin{array}{l}\mathrm{BMoH}- \\
2015 \mathrm{~B}\end{array}$} & \multirow[t]{2}{*}{$\begin{array}{l}\text { TE or } \\
\text { APRI or } \\
\text { FIB-4 }\end{array}$} & \multirow{2}{*}{$\begin{array}{l}\mathrm{LSM} \geq 9.6 \mathrm{kPa} \\
\text { or } \mathrm{APRI} \geq 1.5 \\
\text { or } \\
\text { FIB- } 4 \geq 3.25\end{array}$} & $\begin{array}{l}\text { SOF } 400 \mathrm{mg} \\
{\left[\text { Sovaldi }{ }^{\circledR}-\text { Gilead] }\right.}\end{array}$ & 12 & $\begin{array}{l}1 \\
\text { tablet } \\
\text { daily }\end{array}$ & \multirow{2}{*}{$\begin{array}{l}\text { SOF/SIM for } 12 \text { weeks or } 24 \text { weeks in presence of decompensated } \\
\text { cirrhosis (Child-Pugh B/C). Medical visits at screening, baseline, } \\
\text { W2, W4, W8 and W12. No response-guided therapy or stopping } \\
\text { rules. SVR at W24 }\end{array}$} \\
\hline & & & $\begin{array}{l}\text { SIM } 150 \text { mg } \\
\text { [Olysio - Janssen }{ }^{\circledast}\end{array}$ & 12 & $\begin{array}{l}1 \text { tablet } \\
\text { daily }\end{array}$ & \\
\hline
\end{tabular}

$A P R I$ aspartate-to-platelet index, $B M O H$ Brazilian Ministry of Health, $B O C$ boceprevir, DCV daclastavir, FIB-4 fibrosis-4 score, $k P a$ kilopascal, $L S M$ liver stiffness measurement, PEG-IFN peginterferon, RBV ribavirin, SIM simeprevir, SOF sofosbuvir, SVR sustained-virologic response, TE transient elastography, TEL telaprevir

US\$ 1555.47 per week, respectively. Table 2 summarizes the nominal and PPP-adjusted costs per unit for tests, medical care and drugs used in GT1 HCV patient management.

According to the procedures recommended at the BMoH's guidelines, treatment with PEG-IFN/RBV/first generation PI [BMoH-2013A and B] was the most expensive approach. In those recommendations, patients were treated for 48 weeks by PEG-IFN/RBV and BOC for 44 weeks [total of PPP\$ 71,196.03 (US\$ 39,578.23) per patient] or TEL for 12 weeks [total of PPP\$ $86,250.33$ (US\$ 47,946.92) per patient]. Treatments by new all-oral interferon-free regimens were the least expensive approach: the costs for SOF/DCV [BMoH2015A] and SOF/SIM [BMoH-2015B] were PPP\$ 19,761.72 (US\$ 10,985.90) and PPP\$ 21,590.91 (US\$
12,002.75), respectively (Table 3 ). In the presence of decompensated cirrhosis (Child-Pugh $\mathrm{B} / \mathrm{C}$ ), the necessity to treat with SOF/DCV for 24 weeks leads to an $84 \%$ increase in total costs [PPP\$ 36,597.71 (US\$ 20,345.04)]. In all scenarios, the drug costs were responsible by approximatively $90 \%$ of total cost for treat patients with $\mathrm{CHC}$. The cost with medical visits and blood analysis tests fell by up to $40 \%$ in treatment with DAAs [guidelines BoMH-2015A/B] compared to regimens that used PEG-IFN [guidelines BMoH 2011 and 2013A/B].

\section{Literature overview for efficacy of SOF/DCV and SOF/SIM regimens}

Out of 150 identified articles, 25 references were eligible and 14 real-life cohort studies that reported the efficacy of SOF/DCV $(n=2)$ or SOF/SIM $\pm \operatorname{RBV}(n=12)$ in GT1 
Table 2 Cost per unit and number of procedures for monitoring and injections/tablets for treatment of genotype 1 chronic hepatitis $C$ according to different regimens recommended by the BMoH from 2011 to 2015

\begin{tabular}{|c|c|c|c|c|c|c|c|}
\hline & \multicolumn{2}{|c|}{ Cost per unit } & \multirow{2}{*}{$\begin{array}{l}\text { PEG-RBV } \\
\mathrm{n}\end{array}$} & \multirow{2}{*}{$\begin{array}{l}\text { PEG-RBV/BOC } \\
n\end{array}$} & \multirow{2}{*}{$\begin{array}{l}\text { PEG-RBV/TEL } \\
n\end{array}$} & \multirow{2}{*}{$\begin{array}{l}\text { SOF/DCV } \\
\mathrm{n}\end{array}$} & \multirow{2}{*}{$\begin{array}{l}\text { SOF/SIM } \\
\mathrm{n}\end{array}$} \\
\hline & US\$ & PPP\$ & & & & & \\
\hline Transient elastography & 53.35 & 95.32 & - & 1 & 1 & 1 & 1 \\
\hline Liver biopsy & 482.32 & 834.41 & 1 & - & - & - & - \\
\hline \multicolumn{8}{|l|}{ Blood analysis tests } \\
\hline WBC. RBC and platelet & 10.62 & 19.11 & 15 & 17 & 16 & 6 & 6 \\
\hline Fasting glucose & 4.86 & 8.75 & 6 & 6 & 6 & 6 & 6 \\
\hline BUN/creatinine & 4.84 & 8.70 & 15 & 16 & 15 & 6 & 6 \\
\hline ALT & 4.85 & 8.73 & 15 & 16 & 15 & 6 & 6 \\
\hline AST & 4.86 & 8.74 & 15 & 16 & 15 & 6 & 6 \\
\hline Alkaline phosphatases & 4.77 & 8.58 & 6 & 6 & 6 & 6 & 6 \\
\hline GGT & 4.83 & 8.69 & 6 & 6 & 6 & 6 & 6 \\
\hline Total bilirubin & 4.84 & 8.71 & 6 & 6 & 6 & 6 & 6 \\
\hline Albumin & 5.07 & 9.12 & 6 & 6 & 6 & 6 & 6 \\
\hline INR & 6.68 & 12.02 & 6 & 13 & 14 & 6 & 6 \\
\hline HCV genotype & 156.33 & 281.22 & 1 & 1 & 1 & 1 & 1 \\
\hline HCV-RNA & 87.05 & 156.60 & 8 & 7 & 7 & 2 & 2 \\
\hline TSH & 26.26 & 47.23 & 6 & 6 & 6 & - & - \\
\hline Beta-hCG & 45.52 & 81.88 & 4 & 4 & 4 & 4 & 4 \\
\hline \multicolumn{8}{|l|}{ Medical follow-up } \\
\hline GP's medical visit & 54.54 & 98.11 & 1 & 1 & 1 & 1 & 1 \\
\hline Specialist's medical visit & 62.92 & 113.19 & 14 & 14 & 14 & 5 & 5 \\
\hline Blood sample collection & 37.83 & 68.06 & 15 & 15 & 15 & 6 & 6 \\
\hline Nursing consultation & 31.83 & 57.26 & 5 & 5 & 5 & 4 & 4 \\
\hline \multicolumn{8}{|l|}{ Drugs } \\
\hline PEG-IFN a-2a $180 \mathrm{mcg}$ - SC injection & 360.22 & 648.00 & 48 & 48 & 48 & - & - \\
\hline Ribavirin 250 mg - PO capsule & 1.68 & 3.03 & 1680 & 1680 & 1680 & - & - \\
\hline Boceprevir 200 mg - PO tablet & 4.42 & 7.96 & - & 3612 & - & - & - \\
\hline Telaprevir 375 mg - PO tablet & 48.37 & 87.01 & - & - & 504 & - & - \\
\hline Sofosbuvir 400 mg - PO tablet & 80.54 & 144.89 & - & - & - & 84 & 84 \\
\hline Daclastavir 60 mg - PO tablet & 30.87 & 55.54 & - & - & - & 84 & - \\
\hline Simeprevir 150 mg - PO tablet & 42.98 & 77.32 & - & - & - & - & 84 \\
\hline
\end{tabular}

Costs in United States Dollars (US\$) and with purchasing power parity (PPP\$) on July 2015. Drug costs estimated as the weighted-mean price (http://www.saude.gov.br/bps)

$B O C$ boceprevir, DCV daclastavir, PEG-IFN peginterferon, RBV ribavirin, SIM simeprevir, SOF sofosbuvir, TEL telaprevir, WBC white blood cell, RBC red blood cell, BUN blood urea nitrogen, $A L T$ alanine aminotransferase, AST aspartate aminotransferase, AP alkaline phosphatases, GGT gamma-glutamyl transpeptidase, INR international normalized ratio, $T S H$ thyroid-stimulating hormone, $h C G$ human chorionic gonadotropin, GP general practicioner

Treatment regimens according to BMoH guidelines: PEG-RBV 48w [BMoH-2011]; PEG-RBV (48w)/BOC 44w [BMoH-2013A]; PEG-RBV (48w)/TEL (12w) [BMoH-2013B]; SOF/DCV 12w [BMoH-2015A] and SOF/SIM 12w [BMoH-2015B]

HCV patients were included (Fig. 1). Overall, SVR rates for $\mathrm{SOF} / \mathrm{DCV} \pm \mathrm{RBV}$ and $\mathrm{SOF} / \mathrm{SIM} \pm \mathrm{RBV}$ during 12 weeks ranged from 83 to $98 \%[16,17]$ and from 75 to $96 \%$ [1829], respectively. SVR rates were lower in presence of cirrhosis compared to non-cirrhotic patients (87\% vs $98 \%$ with SOF/DCV [17] and $81 \%$ vs $91 \%$ with SOF/SIM [26]. Higher SVR rates can be achieved in cirrhotic patients when extending SOF/DCV for 24 weeks [17] or adding RBV to SIM/SOF [24]. Pol et al. reported lower SVR rates in GT1a patients compared to GT1b (89\% vs $95 \%$ with SOF/DCV 12 weeks) that might be not significant when extending treatment for 24 weeks (95\% for both) [17]. Similar results were observed in patients treated by SOF/ SIM 12 weeks (83\% for GT1a vs $90 \%$ for GT1b) [26]. Table 4 summarizes SVR rates from real-life cohort studies that analysed the efficacy of SOF/DCV and SOF/SIM regimens in GT1 patients stratified by GT1 subtype and presence or absence of cirrhosis or previous treatment. 
Table 3 Monitoring and drugs costs (US\$ and PPP-adjusted US\$) for treatment of genotype-1 patient with chronic hepatitis C according to different regimens recommended by the BMoH from 2011 to 2015

\begin{tabular}{|c|c|c|c|c|c|c|c|c|c|c|}
\hline & \multicolumn{2}{|l|}{ PEG-RBV } & \multicolumn{2}{|c|}{ PEG-RBV/BOC } & \multicolumn{2}{|c|}{ PEG-RBV/TEL } & \multicolumn{2}{|l|}{ SOF/DCV } & \multicolumn{2}{|l|}{ SOF/SIM } \\
\hline & US\$ & PPP\$ & US\$ & PPP\$ & US\$ & PPP\$ & US\$ & PPP\$ & US\$ & PPP\$ \\
\hline TE & - & - & 53.35 & 95.32 & 53.35 & 95.32 & 53.35 & 95.32 & 53.35 & 95.32 \\
\hline Liver biopsy & 482.32 & 834.41 & - & - & - & - & - & - & - & - \\
\hline Total & 482.32 & 834.41 & 53.35 & 95.32 & 53.35 & 95.32 & 53.35 & 95.32 & 53.35 & 95.32 \\
\hline \multicolumn{11}{|l|}{ Blood analysis tests } \\
\hline WBC. RBC and platelet & 159.39 & 286.72 & 180.64 & 324.95 & 170.02 & 305.84 & 63.76 & 114.69 & 63.76 & 114.69 \\
\hline Fasting glucose & 29.20 & 52.52 & 29.20 & 52.52 & 29.20 & 52.52 & 29.20 & 52.52 & 29.20 & 52.52 \\
\hline BUN/creatinine & 72.58 & 130.56 & 77.41 & 139.26 & 72.58 & 130.56 & 29.03 & 52.22 & 29.03 & 52.22 \\
\hline ALT & 72.78 & 130.92 & 77.63 & 139.65 & 72.78 & 130.92 & 29.11 & 52.37 & 29.11 & 52.37 \\
\hline AST & 72.91 & 131.16 & 77.78 & 139.91 & 72.91 & 131.16 & 29.17 & 52.47 & 29.17 & 52.47 \\
\hline$A P$ & 28.61 & 51.46 & 28.61 & 51.46 & 28.61 & 51.46 & 28.61 & 51.46 & 28.61 & 51.46 \\
\hline GGT & 28.99 & 52.15 & 28.99 & 52.15 & 28.99 & 52.15 & 28.99 & 52.15 & 28.99 & 52.15 \\
\hline Total bilirubin & 29.04 & 52.24 & 29.04 & 52.24 & 29.04 & 52.24 & 29.04 & 52.24 & 29.04 & 52.24 \\
\hline Albumin & 30.44 & 54.75 & 30.44 & 54.75 & 30.44 & 54.75 & 30.44 & 54.75 & 30.44 & 54.75 \\
\hline INR & 40.10 & 72.14 & 86.89 & 156.30 & 93.57 & 168.32 & 40.10 & 72.14 & 40.10 & 72.14 \\
\hline HCV genotype & 156.33 & 281.22 & 156.33 & 281.22 & 156.33 & 281.22 & 156.33 & 281.22 & 156.33 & 281.22 \\
\hline HCV-RNA & 696.43 & 1252.80 & 609.38 & 1096.20 & 609.38 & 1096.20 & 174.11 & 313.20 & 174.11 & 313.20 \\
\hline TSH & 157.55 & 283.41 & 157.55 & 283.41 & 157.55 & 283.41 & & - & & - \\
\hline Beta-hCG & 182.07 & 327.53 & 182.07 & 327.53 & 182.07 & 327.53 & 182.07 & 327.53 & 182.07 & 327.53 \\
\hline Total & 1756.41 & 3159.58 & 1751.94 & 3151.54 & 1733.45 & 3118.27 & 849.94 & 1528.95 & 849.94 & 1528.95 \\
\hline \multicolumn{11}{|l|}{ Medical follow-up } \\
\hline GP's medical visit & 54.54 & 98.11 & 54.54 & 98.11 & 54.54 & 98.11 & 54.54 & 98.11 & 54.54 & 98.11 \\
\hline Specialist's visit & 880.95 & 1584.73 & 880.95 & 1584.73 & 880.95 & 1584.73 & 314.62 & 565.97 & 314.62 & 565.97 \\
\hline Blood sample & 567.52 & 1020.90 & 567.52 & 1020.90 & 567.52 & 1020.90 & 227.01 & 408.36 & 227.01 & 408.36 \\
\hline Nursing consultation & 159.15 & 286.29 & 159.15 & 286.29 & 159.15 & 286.29 & 127.32 & 229.03 & 127.32 & 229.03 \\
\hline Total & 1662.15 & 2990.02 & 1662.15 & 2990.02 & 1662.15 & 2990.02 & 723.49 & 1301.47 & 723.49 & 1301.47 \\
\hline \multicolumn{11}{|l|}{ Drugs } \\
\hline PEG-IFN $180 \mathrm{mcg}$ & $17,290.59$ & $31,103.78$ & $17,290.59$ & $31,103.78$ & $17,290.59$ & $31,103.78$ & - & - & - & - \\
\hline Ribavirin 250 mg & 2828.69 & 5088.49 & 2828.69 & 5088.49 & 2828.69 & 5088.49 & - & - & - & - \\
\hline Boceprevir 200 mg & - & - & $15,991.51$ & $28,766.88$ & - & - & - & - & - & - \\
\hline Telaprevir 375 mg & - & - & - & - & $24,378.69$ & $43,854.45$ & - & - & - & - \\
\hline Sofosbuvir 400 mg & - & - & - & - & - & - & 6765.68 & $12,170.68$ & 6765.68 & $12,170.68$ \\
\hline Daclastavir 60 mg & - & - & - & - & - & - & 2593.44 & 4665.30 & - & - \\
\hline Simeprevir 150 mg & - & - & - & - & - & - & - & - & 3610.29 & 6494.49 \\
\hline Total & $20,119.28$ & $36,192.27$ & $36,110.79$ & $64,959.15$ & $44,497.97$ & $80,046.72$ & 9359.12 & $16,835.98$ & $10,375.97$ & $18,665.17$ \\
\hline TOTAL COST & $24,020.16$ & $43,176.28$ & $39,578.23$ & $71,196.03$ & $47,946.92$ & $86,250.33$ & $10,985.90$ & $19,761.72$ & $12,002.75$ & $21,590.91$ \\
\hline
\end{tabular}

Costs in United States Dollars (US\$) and with purchasing power parity (PPP\$) on July 2015. Drug costs estimated as the weighted-mean price (http://www.saude.gov.br/bps)

PEG-IFN peginterferon, WBC white blood cell, RBC red blood cell, BUN blood urea nitrogen, $A L T$ alanine aminotransferase, AST aspartate aminotransferase, AP alkaline phosphatases, GGT gamma-glutamyl transpeptidase, INR international normalized ratio, TSH thyroid-stimulating hormone, $h C G$ human chorionic gonadotropin, GP general practicioner

Treatment regimens according to BMoH guidelines: PEG-RBV 48w [BMoH-2011]; PEG-RBV (48w)/BOC 44w [BMoH-2013A]; PEG-RBV (48w)/TEL (12w) [BMoH-2013B]; SOF/DCV 12w [BMoH-2015A] and SOF/SIM 12w [BMoH-2015B] 

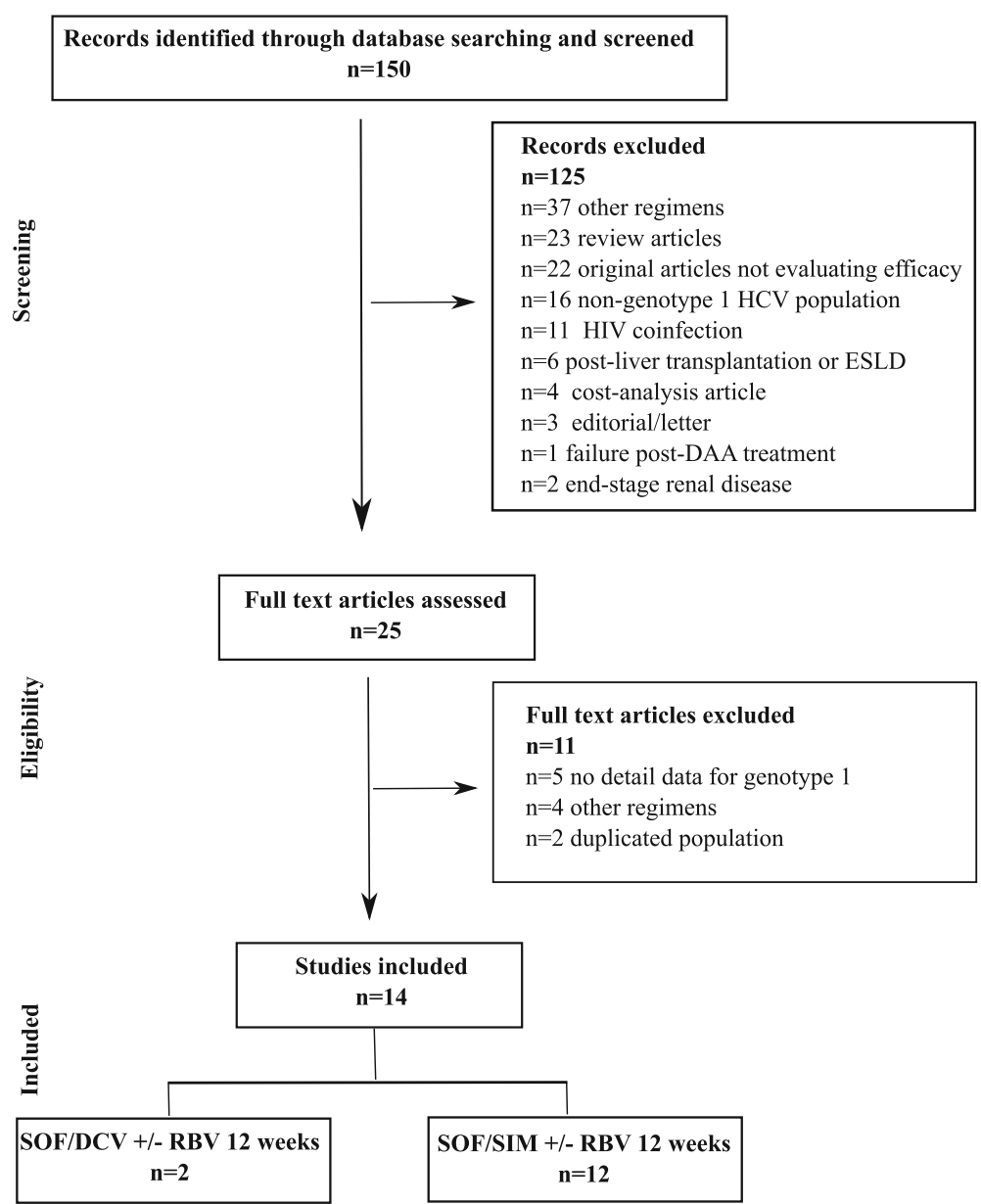

Fig. 1 Flow-chart of study selection for the literature overview

\section{Discussion}

The present study highlighted that GT1 HCV treatment by effective DAAs might be less expensive than interferon-based regimens in a low-to-middle income country, mainly due to price negotiation between health authorities and pharmaceutical companies and lower costs with monitoring in the all-oral interferon-free treatments.

The cost of TE was increased due to the very high interest rates used in Brazil [30,31] and higher expenses with personnel at INI/FIOCRUZ. TE could be less costly in countries with lower opportunity cost of public funds and in those where nurses perform the exams. Liver fibrosis staging by TE seems to be cost-effective compared to liver biopsy and it was priced from US\$ 21 to US\$ 73 in the United Kingdom and up to US\$ 145 in the United States [4].

$\mathrm{HCV}$ eradication has been associated with a financial burden, especially due to high DAAs costs which are extremely variable worldwide [6]. An analysis of for a 12-week SOF course price (PPP-adjusted) in 30 countries described that Poland (PPP\$ 101,063.00), Turkey (PPP\$ 91,339) and United States (PPP\$ 64,680) were the top three ranking countries with the most expensive prices. On the other hand, India (PPP\$ 1861), Mongolia (PPP\$ 2604) and Egypt (PPP\$ 3117) were the countries with the least expensive price for SOF-therapy [32]. In 36 clinical sites in USA, real-world costs for PEG-IFN/RBV/BOC 48 weeks, PEG-IFN/TEL 48 weeks and SOF/SIM 12 weeks were US\$ 57,960; US\$ 113,400; US\$ 180,600; respectively. In addition, the mean cost per SVR in all-oral interferon-free was higher in patients with advanced fibrosis (US\$ 167,467 for F3F4 vs 116,579 for F0F1F2) [33]. However, both studies only included cost of HCV medications, not adjunctive medications, hospital costs, or projected medical costs. Data from Europe and Canada reported a total cost [mean $(95 \% \mathrm{CI})]$ of $€ 14,559(13,323-15,836)$ per patient and $€ 38,514(35,244-41,892)$ per SVR for IFN-based regimens considering treatment, monitoring and complications [34]. The price disparities among countries might be explained partly by the pharmaceutical price-setting 
Table 4 Real-life cohort studies that evaluated efficacy (SVR12) of Sofosbuvir/Daclastavir (SOF/DCV) or Sofosbuvir/Simeprevir (SOF/ SIM) regimens for treatment of HCV mono-infected genotype-1 patients

\begin{tabular}{|c|c|c|c|c|c|c|c|c|c|c|c|c|c|c|c|c|c|}
\hline \multirow[t]{2}{*}{ Authors } & \multirow[t]{2}{*}{ Year } & \multirow[t]{2}{*}{ Local } & \multirow[t]{2}{*}{ Regimen } & \multicolumn{2}{|c|}{ Overall } & \multicolumn{2}{|c|}{ Non-cirrhosis } & \multicolumn{2}{|c|}{ Cirrhosis } & \multicolumn{2}{|c|}{ Naive } & \multicolumn{2}{|c|}{ Experimented } & \multicolumn{2}{|c|}{ GT 1a } & \multicolumn{2}{|c|}{ GT 1b } \\
\hline & & & & $\mathrm{n}$ & SVR & $\mathrm{n}$ & SVR & $\mathrm{n}$ & SVR & $\mathrm{n}$ & SVR & $\mathrm{n}$ & SVR & $\mathrm{n}$ & SVR & $\mathrm{n}$ & SVR \\
\hline \multicolumn{18}{|c|}{ Sofosbuvir (400 mg)/Daclastavir (60 mg) regimen studies } \\
\hline \multirow[t]{2}{*}{ Pol et al. [17] } & 2017 & France & SOF/DCV 12w & 160 & $92 \%$ & 66 & $98 \%$ & 94 & $87 \%$ & 64 & $88 \%$ & 96 & $95 \%$ & 73 & $95 \%$ & 83 & $89 \%$ \\
\hline & & & SOF/DCV 24w & 439 & $95 \%$ & 97 & $97 \%$ & 342 & $94 \%$ & 48 & $85 \%$ & 391 & $96 \%$ & 210 & $95 \%$ & 209 & $95 \%$ \\
\hline Welzel et al. [16] & 2016 & Europe & SOF/DCV \pm RBV $12 \mathrm{w}$ & 319 & $98 \%$ & - & - & - & - & - & - & - & - & 151 & $99 \%$ & 155 & $97 \%$ \\
\hline \multicolumn{18}{|c|}{ Sofosbuvir (400 mg)/Simeprevir (150 mg) regimen studies } \\
\hline Marino et al. [[28] & 2017 & Spain & $\mathrm{SOF} / \mathrm{SIM} \pm \mathrm{RBV} 12 \mathrm{w}$ & 835 & $92 \%$ & - & - & 835 & $92 \%$ & - & - & - & - & - & - & - & - \\
\hline Ramos et al. [29] & 2017 & Spain & SOF/SIM 12w & 179 & $93 \%$ & - & - & - & - & - & - & - & - & - & - & - & - \\
\hline Chang et al. [27] & 2017 & USA & SOF/SIM 12w & 21 & $91 \%$ & - & - & - & - & - & - & - & - & - & - & - & - \\
\hline Sulkowski et al. [26] & 2016 & USA & SOF/SIM 12w & 639 & $85 \%$ & 272 & $91 \%$ & 367 & $81 \%$ & 65 & - & - & - & 371 & $83 \%$ & 185 & $90 \%$ \\
\hline Lutchtman et al. [25] & 2016 & USA & SOF/SIM 12w & 148 & $82 \%$ & 43 & $88 \%$ & 193 & $78 \%$ & 62 & $87 \%$ & 86 & $78 \%$ & 94 & $79 \%$ & 42 & $93 \%$ \\
\hline Pellicelli et al. [24] & 2016 & Italy & SOF/SIM/RBV 12W & 270 & $96 \%$ & - & - & 270 & $96 \%$ & - & - & - & - & - & - & - & - \\
\hline Roytman et al. [23] & 2016 & USA & SOF/SIM 12W & 138 & $89 \%$ & 43 & $93 \%$ & 95 & $87 \%$ & 76 & $87 \%$ & 55 & $92 \%$ & - & - & - & - \\
\hline Thornton et al. [22] & 2016 & USA & SOF/SIM 12w & 114 & $89 \%$ & - & - & - & - & - & - & - & - & - & - & - & - \\
\hline Jayasekera et al. [19] & 2016 & USA & SOF/SIM 12w & 35 & $86 \%$ & 13 & $92 \%$ & 22 & $82 \%$ & 7 & $86 \%$ & 28 & $86 \%$ & - & - & - & - \\
\hline Pillail et al. [20] & 2016 & USA & $\mathrm{SOF} / \mathrm{SIM} \pm \mathrm{RBV} 12 \mathrm{w}$ & 113 & $84 \%$ & 23 & $91 \%$ & 90 & $82 \%$ & 57 & $89 \%$ & 56 & $79 \%$ & - & - & - & - \\
\hline Barron et al. [21] & 2016 & USA & $\mathrm{SOF} / \mathrm{SIM} \pm \mathrm{RBV} 12 \mathrm{w}$ & 35 & $83 \%$ & - & - & - & - & - & - & - & - & - & - & - & - \\
\hline Backus et al. [18] & 2015 & USA & SOF/SIM 12w & 1130 & $75 \%$ & 664 & $81 \%$ & 462 & $68 \%$ & 699 & $78 \%$ & 431 & $71 \%$ & 632 & $73 \%$ & 366 & $80 \%$ \\
\hline
\end{tabular}

$D C V$ daclastavir, GT genotype, SIM simeprevir, SOF sofosbuvir, SVR sustained virological response, $w$ week

policies used in different countries and negotiation between health authorities and pharmaceutical companies. Some countries set prices according to explicit costeffectiveness thresholds based on gross domestic product (GDP) per capita resulting in high prices without consideration of budget impact [35]. Therefore, DAA treatment costs are lower in Brazil compared to others countries mainly due to intense negotiation of $\mathrm{BMoH}$ with pharmaceutical companies resulting in more than 90\% discount over international prices for SOF, DCV and SIM [9].

In the present study, the reduced price for DAAs and shorten treatment duration might explain the lower total cost for $\mathrm{HCV}$ eradication with all-oral interferonfree compared to IFN-based regimens. Weekly prices for PEG-IFN (US\$ 360 or PPP\$ 648), BOC (US\$ 371 or PPP\$ 672) and TEL (US\$ 2030 or PPP\$ 3654) were relatively high and longer duration treatment with IFNregimens led to higher costs with monitoring and medical visits (mean cost of PPP\$ 6133.15 for IFN-based vs PPP\$ 2830.42 for interferon-free regimens). In addition, adverse events are more frequent in IFN-based regimens than DAA treatment leading to higher costs. Previous studies reported that extra medical visits and drugs used for adverse events treatment, such as epoetin- $\alpha$ or filgrastim, lead to an increase in costs for treatment with IFN-based regimens with first generation PI (BOC/TEL) [36]. Costs per SVR might have 2- fold increase for patients with thrombocytopenia compared to those with normal platelet count when treated by IFN-based regimens [€50,907 (95\% CI, 44,151$59,612)$ vs $€ 26,105(23,068-29,296)]$ [34].

Data from real-world cohort studies have been showing higher SVR rates by 12-week course of SOF/DCV (up to 92\%) [17] and SOF/SIM regimens (up to 85\%) [26] compared to IFN-based regimens (up to $40 \%$ in cirrhotic patients treated by PEG-IFN/RBV/BOC or TEL) [37]. However, presence of cirrhosis and GT subtype 1a might lead to lower SVR rates (Table 4). The extension of SOF/DCV for 24 weeks might be used to achieve higher SVR rates in cirrhotic patients [17]. In GT1 HCV infected patients, treatment using DAAs has been considered as cost-effective [38] and further analysis are needed to evaluate whether this approach is cost-saving [39]. In addition, treatment by SOF/DCV or SOF/SIM result in less medical visits and blood analysis tests for monitoring, lower rates of adverse events, no need of subcutaneous injection and lower intake of tablets/day compared to IFN-regimens. However, presence of decompensated cirrhosis highly increase the cost of treatment [from PPP\$ 19,761.72to 36, 597.71 (from US\$ $10,985.9$ to $20,345.04$ ] due to extension of SOF/DCV regimen for 24 weeks. Despite, the lack of costeffectiveness studies in Brazil, given the fact that DAAregimens are more effective compared to IFN-regimens and total cost for $\mathrm{HCV}$ treatment with all-oral 
interferon-free drugs is lower than IFN-based regimens, we could hypothesize that treatment with DAAs might be dominant in this country. Considering local costs and SVR rates reported in international real-life cohorts, the cost per SVR in Brazil could range from 20 to 30 thousand PPP\$. Early viral kinetics might be used to individualize duration of DAA therapy decreasing costs by up to $20 \%$ [40]. On the other hand, treatment of patients with advanced fibrosis/compensated cirrhosis for 12 weeks, as recommended by the $\mathrm{BMoH}$ guideline, might lead to relative high rates of DAA failure or resistance and increased future costs for re-treatment. The addition of RBV (1250 mg PO daily) to SOF/SIM or SOF/DCV does not highly increase the cost of treatment due to local generic production and low price (US\$ 706 or PPP\$ 1273 per patient per 12 week duration). In Brazil, the prevalence of GT $1 \mathrm{~b}$ seems to slightly higher than 1a (55\% vs $45 \%)$ [41]. Considering our HCV prevalence and costs described in the present study, the financial burden could be up to PPP\$ 21 billion (or US\$ 12 billion) to treat all GT1 HCV infected people independently of fibrosis stage in Brazil. This amount represents approximately $13 \%$ of the general government expenditure on health in Brazil during 2014 [Health expenditure series. World Health Organization, available at: http://apps.who.int/nha/database/Select/Indicators/en; accessed at March 2017].

The main limitations of the present study are the lack of primary data on patient's follow-up and costs of treatment for non-GT1 patients and new generation of interferon-free regimens. Presence of adverse events might lead to increased costs for medical care and low treatment adherence might result in higher costs and higher costs per SVR than our estimates, due to nonresponse/relapse. However, primary data on DAAtreatment remain scarce in Brazil because the strategy from BMoH for universal access to SOF, DCV and SIM has been recently implemented. We estimated the costs of an ideal follow-up for standard treatment of GT1, the most prevalent genotype, $\mathrm{HCV}$ patients according to recent $\mathrm{BMoH}$ 's recommendations. We are aware that treatment with fixed dose one-tablet daily of $\mathrm{SOF} /$ ledispavir $(400 / 90 \mathrm{mg})$ or $\mathrm{SOF} /$ velpatasvir $(400 / 100 \mathrm{mg})$ for 12 weeks has been recommended as first-line therapies for GT1 patients with or without cirrhosis [42]. These patients can also be treated with the fixed-dose combination of ombitasvir $(12.5 \mathrm{mg})$, paritaprevir $(75 \mathrm{mg})$ and ritonavir $(50 \mathrm{mg})$ in one single tablet and dasabuvir (250 mg) [PTV/r/OMV + DSV regimen] or the fixed dose combination of grazoprevir $(100 \mathrm{mg})$ and elbasvir $(50 \mathrm{mg})$ in a single tablet once-daily [42]. However, these drug combinations are not currently available in Brazil for cost estimation. Another major limitation might be the fact that we evaluated the costs for $\mathrm{HCV}$ treatment in a short-term horizon and without prospective followup to evaluate whether DAAs treatment are cost-saving compared to PEG-IFN regimens.

The main strengths of this analysis are the PPPadjustment for costs, the estimation of "real-price" for $\mathrm{HCV}$-drugs and those related to monitoring/medical care accounting for the economic costs of public funds in medical investments. PPP adjustment is essential to allow comparison of prices of goods and services across countries. For high-income countries, PPP adjustment results in lower prices. In contrast, countries with weaker purchasing power, such as Brazil, have a significant increase in prices with PPP adjustment [43]. In the present study, the PPP-adjustment led to an increase in 1.8 fold in US\$ costs. The cost for each drug were defined as the weighted-mean price of all HCV-drugs bought by the $\mathrm{BMoH}$ in 2015 and registered in webbased and open-access platform. In addition, we analysed micro-costs of all procedures recommended pre, during and post-HCV treatment to allow the estimation of medical care financial burden beyond drug costs.

\section{Conclusions}

In conclusion, therapies with all-oral interferon-free, such as SOF/DCV and SOF/SIM for 12 weeks, have a high efficacy and need shorter treatment duration leading to lower costs for monitoring and medical care compared to IFNbased regimens in Brazil where DAAs had more than $90 \%$ discount over international prices. HCV treatment by IFN-free regimens will probably be associated with high financial impact worldwide. However, reduced DAA prices due to negotiation between health authorities and pharmaceutical companies can turn HCV treatment into an accessible strategy to $\mathrm{HCV}$ eradication.

\section{Additional file}

Additional file 1: Micro-costing analysis of guideline-based treatment by direct-acting agents: the real-life case of hepatitis $C$ management in Brazil. (DOCX 28 kb)

\footnotetext{
Abbreviations

ABC: Activity based costing; ALT: Alanine aminotransferase; APRI: Aspartateto-platelet ratio index; AST: Aspartate aminotransferase; $\mathrm{BMoH}$ : Brazilian Ministry of Health; BOC: Boceprevir; BRL: Brazilian Real; CHC: Chronic hepatitis C; DAA: Direct-acting antiviral drugs; DCV: Daclastavir; FIB-4: Fibrosis-4 score; GDP: Gross domestic product; GT1: Genotype 1; HCV: Hepatitis C virus; PEGIFN: PEG-interferon; PPP\$: Purchasing power parity Dollars; RBV: Ribavirin; SELIC: Special System for Settlement and Custody; SIM: Simeprevir; SOF: Sofosbuvir; SUS: Sistema Único de Saúde; SVR: Sustained virological response; TE: Transient elastography; TEL: Telaprevir; US\$: United States Dollars
} 


\section{Funding}

This work was supported by funding from Newton Fund RCUK-CONFAP Research Partnerships call, project "Brazil-UK collaboration in Health Technology Assessment of diagnostic strategies for infectious diseases" from Fundação Carlos Chagas Filho de Amparo à Pesquisa do Estado do Rio de Janeiro (FAPERJ) under grant E-26/170.021/2015 [Brazil for RC] and Medical Research Council (MRC) grant reference MR/M026515/1 [UK for CH]. The funders had no role in study design, data collection and analysis, decision to publish, or preparation of the manuscript.

\section{Availability of data and materials}

All data from the current study were reported in the manuscript, tables and supplementary material. The datasets generated and/or analyzed during the current study are available in a web-based public domain: https://lapecos.ipec.fiocruz.br/. In addition, data are available upon request to Hugo Perazzo, the corresponding author, and with permission of the Ethical Committee of Instituto Nacional de Infectologia Evandro Chagas - Fundação Oswaldo Cruz, Rio de Janeiro (RJ), Brazil.

\section{Authors' contributions}

HP: study concept and design; interpretation of data; drafting and critical revision of the manuscript. MJJ: study concept and design; acquisition of data; analysis and interpretation of data; statistical analysis and critical revision of the manuscript. JCS, AMA, PSS: acquisition of data; analysis and interpretation of data; statistical analysis and critical revision of the manuscript. CR, VGV, RMM, RA: interpretation of data and critical revision of the manuscript. $\mathrm{CH}$ and RC: study concept and design; study supervision, interpretation of data and critical revision of the manuscript. All authors read and approved the final manuscript.

\section{Ethics approval and consent to participate}

This study did not analyzed primary data from patients or participants. The study was approved with waiver of informed consent by the Ethical Committee from Instituto Nacional de Infectologia Evandro Chagas - Fundação Oswaldo Cruz (IRB n 51,736,815.3.0000.5262).

\section{Consent for publication}

Not applicable.

\section{Competing interests}

The authors declare that they have no competing interests.

\section{Publisher's Note}

Springer Nature remains neutral with regard to jurisdictional claims in published maps and institutional affiliations.

\section{Author details}

${ }^{1}$ Fundação Oswaldo Cruz, FIOCRUZ, Instituto Nacional de Infectologia Evandro Chagas, INI, Laboratório de Pesquisa Clínica em DST e AIDS, LAPCLIN-AIDS, Rio de Janeiro, Brazil. Fundação Oswaldo Cruz, FIOCRUZ, Instituto Nacional de Infectologia Evandro Chagas, INI, Laboratório de Pesquisa em Economia das Organizações de Saúde, LAPECOS, Rio de Janeiro, Brazil. 'Fundação Oswaldo Cruz, FIOCRUZ, Instituto Nacional de Infectologia Evandro Chagas, INI, Plataforma de Pesquisa Clínica, Rio de Janeiro, Brazil. ${ }^{4}$ Fundação Oswaldo Cruz, FIOCRUZ, Centro de Desenvolvimento Tecnológico em Saúde, CDTS, Rio de Janeiro, Brazil. ${ }^{5}$ University of Exeter Medical School, UEMS, Evidence Synthesis \& Modelling for Health Improvement, ESMI, Exeter, UK. ${ }^{6}$ Universidade Federal do Estado do Rio de Janeiro, UNIRIO, Instituto de Saúde Coletiva, ISC, Rio de Janeiro, Brazil.

\section{Received: 6 April 2017 Accepted: 15 November 2017}

Published online: 23 November 2017

\section{References}

1. Westbrook RH, Dusheiko G. Natural history of hepatitis C. J Hepatol. 2014; 61(1 Suppl):S58-68.

2. Nahon P, Bourcier V, Layese R, Audureau E, Cagnot C, Marcellin P, Guyader $D$, Fontaine $H$, Larrey D, De Ledinghen $V$, et al. Eradication of hepatitis C virus infection in patients with cirrhosis reduces risk of liver and non-liver complications. Gastroenterology. 2017;152(1):142-56.
3. Bota S, Herkner H, Sporea I, Salzl P, Sirli R, Neghina AM, Peck-Radosavljevic M. Meta-analysis: ARFI elastography versus transient elastography for the evaluation of liver fibrosis. Liver Int. 2013;33(8):1138-47.

4. van Katwyk S, Coyle D, Cooper C, Pussegoda K, Cameron C, Skidmore B, Brener S, Moher D, Thavorn K. Transient elastography for the diagnosis of liver fibrosis: a systematic review of economic evaluations. Liver Int. 2017;37(6):851-61.

5. Flisiak R, Pogorzelska J, Flisiak-Jackiewicz M. Hepatitis C: efficacy and safety in real life. Liver Int. 2017;1:26-32.

6. Ward T, Gordon J, Bennett H, Webster S, Sugrue D, Jones B, Brenner M, McEwan P. Tackling the burden of the hepatitis $C$ virus in the UK: characterizing and assessing the clinical and economic consequences. Public Health. 2016;141:42-51.

7. Linas BP. Understanding cost and value in hepatitis C therapy. Top Antivir Med. 2016;24(2):93-7.

8. Jacobs JC, Barnett PG. Emergent challenges in determining costs for economic evaluations. PharmacoEconomics. 2017;35(2):129-39.

9. Mesquita F, Santos ME, Benzaken A, Correa RG, Cattapan E, Sereno LS, Naveira MC. The Brazilian comprehensive response to hepatitis C: from strategic thinking to access to interferon-free therapy. BMC Public Health. 2016;16(1):1132

10. Vigani AG, Pavan MH, Tozzo R, Goncales ES, Feltrin A, Fais VC, Lazarini MS, Goncales NS, Goncales FL Jr. Comparative study of patients with chronic hepatitis $C$ virus infection due to genotypes 1 and 3 referred for treatment in southeast Brazil. BMC Infect Dis. 2008;8:164.

11. Fernandes FF, Ferraz ML, Andrade LE, Dellavance A, Terra C, Pereira G, Pereira JL, Campos F, Figueiredo F, Perez RM. Enhanced liver fibrosis panel as a predictor of liver fibrosis in chronic hepatitis C patients. J Clin Gastroenterol. 2015;49(3):235-41.

12. Brasil. Guideline for management and treatment of chronic hepatitis $C$ and its co-infections [Protocolo Clinico e Diretrizes Terapêuticas para Tratamento da Hepatite viral Crônica C e co-infecções]. Brasilia: Brazilian Ministry of Health [Ministerio da Saude do Brasil]; 2015. http://portalarquivos.saude.gov. br/images/pdf/2015/julho/29/PCDT-FINAL-HEPATITE-C-FINAL-01.pdf [Accessed on 27 Mar 2017]

13. Udpa S. Activity-based costing for hospitals. Health Care Manag Rev. 1996 21(3):83-96.

14. Brasil. Guideline for management and treatment of chronic hepatitis $C$ and its co-infections [Protocolo Clinico e Diretrizes Terapêuticas para Tratamento da Hepatite viral Crônica C e co-infecções]. Brasilia: Brazilian Ministry of Health [Ministerio da Saude do Brasil]; 2011. http://bvsms.saude.gov.br/bvs/ publicacoes/protocolos_diretrizes_hepatite_viral_c_coinfeccoes.pdf [Accessed on 27 Mar 2017]

15. Brasil. Guideline for management and treatment of chronic hepatitis $C$ and its co-infections [Protocolo Clinico e Diretrizes Terapêuticas para Tratamento da Hepatite viral Crônica C e co-infecções]. Brasilia: Brazilian Ministry of Health [Ministerio da Saude do Brasil]; 2013. http://www.funed.mg.gov.br/ wp-content/uploads/2011/07/suplemento_1_protocolo_hep_c.pdf [Accessed on 27 Mar 2017]

16. Welzel TM, Petersen J, Herzer K, Ferenci P, Gschwantler M, Wedemeyer H, Berg T, Spengler U, Weiland O, van der Valk M, et al. Daclatasvir plus sofosbuvir, with or without ribavirin, achieved high sustained virological response rates in patients with $\mathrm{HCV}$ infection and advanced liver disease in a real-world cohort. Gut. 2016:65(11):1861-70.

17. Pol S, Bourliere M, Lucier S, Hezode C, Dorival C, Larrey D, Bronowicki JP, Ledinghen VD, Zoulim F, Tran A, et al. Safety and efficacy of daclatasvir-sofosbuvir in HCV genotype 1-mono-infected patients. J Hepatol. 2017;66(1):39-47.

18. Backus LI, Belperio PS, Shahoumian TA, Loomis TP, Mole LA. Effectiveness of sofosbuvir-based regimens in genotype 1 and 2 hepatitis $C$ virus infection in 4026 U.S. veterans. Aliment Pharmacol Ther. 2015;42(5):559-73.

19. Jayasekera CR, Perumpail RB, Chao DT, Pham EA, Aggarwal A, Wong RJ, Ahmed A. Task-shifting: an approach to decentralized hepatitis C treatment in medically underserved areas. Dig Dis Sci. 2015:60(12):3552-7.

20. Pillai AA, Wedd J, Norvell JP, Parekh S, Cheng N, Young N, Spivey JR, Ford R Simeprevir and Sofosbuvir (SMV-SOF) for 12 weeks for the treatment of chronic hepatitis $C$ genotype 1 infection: a real world (transplant) Hepatology practice experience. Am J Gastroenterol. 2016:111(2):250-60.

21. Barron J, Xie Y, Wu SJ, White J, Singer J, Tulsi B, Rosenberg A. Treatment of chronic hepatitis $C$ infection with Sofosbuvir-based regimens in a commercially insured patient population. Am Health Drug Benefits. 2016;9(6):327-35.

22. Thornton K, Deming P, Manch RA, Moore A, Kohli A, Gish R, Sussman NL, Khaderi S, Scott J, Mera J, et al. Is response guided therapy dead? Low cure 
rates in patients with detectable hepatitis $C$ virus at week 4 of treatment. Hepatol Int. 2016;10(4):624-31.

23. Roytman M, Ramkissoon R, Wu C, Hong L, Trujillo R, Huddleston L, Poerzgen $P$, Seto T, Wong L, Tsai N. Examining the clinical course of genotype 1 chronic hepatitis $\mathrm{C}$ patients treated with the cosmos regimen: including patients with advanced liver disease and east Asian ancestry. Hepatol Int. 2016;10(4):616-23.

24. Pellicelli AM, Pace Palitti V, Vignally P, Ceccherini-Silberstein F, Siciliano M, Giannelli V, Moretti A, Tarquini P, Scifo G, Messina V, et al. Efficacy and safety of sofosbuvir/simeprevir plus flat dose ribavirin in genotype 1 elderly cirrhotic patients: a real-life study. Liver Int. 2017;37(5):653-61.

25. Lutchman G, Nguyen NH, Chang CY, Ahmed A, Daugherty T, Garcia G, Kumari R, Gupta S, Doshi D, Nguyen MH. Effectiveness and tolerability of simeprevir and sofosbuvir in nontransplant and post-liver transplant patients with hepatitis C genotype 1. Aliment Pharmacol Ther. 2016;44(7): 738-46.

26. Sulkowski MS, Vargas HE, Di Bisceglie AM, Kuo A, Reddy KR, Lim JK, Morelli G, Darling JM, Feld JJ, Brown RS, et al. Effectiveness of Simeprevir plus Sofosbuvir, with or without Ribavirin, in real-world patients with HCV genotype 1 infection. Gastroenterology. 2016;150(2):419-29.

27. Chang CY, Nguyen P, Le A, Zhao C, Ahmed A, Daugherty T, Garcia G, Lutchman G, Kumari R, Nguyen MH. Real-world experience with interferonfree, direct acting antiviral therapies in Asian Americans with chronic hepatitis C and advanced liver disease. Medicine (Baltimore). 2017;96(6): e6128.

28. Marino Z, Pascasio-Acevedo JM, Gallego A, Diago M, Baliellas C, Morillas R, Prieto M, Moreno JM, Sanchez-Antolin G, Vergara M et al. High efficacy of Sofosbuvir plus Simeprevir in a large cohort of Spanish cirrhotic patients infected with genotypes 1 and 4. Liver Int. 2017. doi: https://doi.org/10. 1111/liv.13470. [Epub ahead of print].

29. Ramos H, Linares P, Badia E, Martin I, Gomez J, Almohalla C, Jorquera F, Calvo S, Garcia I, Conde P, et al. Interferon-free treatments in patients with hepatitis C genotype 1-4 infections in a real-world setting. World J Gastrointest Pharmacol Ther. 2017;8(2):137-46.

30. de Lucinda CR, Vieira RL. Interest rates and informational issues in the credit market: experimental evidence from Brazil. World Dev. 2014;59:47-58.

31. Modenesi AD, Modenesi RL. Development conventions: theory and the case of Brazil in the latter half of the twentieth century. J Post Keynesian Econ. 2015;38(1):131-61.

32. lyengar S, Tay-Teo K, Vogler S, Beyer P, Wiktor S, de Joncheere K, Hill S Prices, costs, and affordability of new medicines for hepatitis $\mathrm{C}$ in 30 countries: an economic analysis. PLoS Med. 2016;13(5):e1002032.

33. Bach TA, Zaiken $\mathrm{K}$. Real-world drug costs of treating hepatitis $\mathrm{C}$ genotypes 1-4 with direct-acting Antivirals: initiating treatment at fibrosis $0-2$ and 3-4. J Manag Care Spec Pharm. 2016;22(12):1437-45.

34. Maan R, Zaim R, van der Meer AJ, Feld JJ, Wedemeyer H, Dufour JF, Lammert F, Manns MP, Zeuzem S, Hansen BE, et al. Real-world medical costs of antiviral therapy among patients with chronic HCV infection and advanced hepatic fibrosis. J Gastroenterol Hepatol. 2016;31(11):1851-9.

35. Jakubiak-Lasocka J, Jakubczyk M. Cost-effectiveness versus cost-utility analyses: what are the motives behind using each and how do their results differ? - a polish example. Value Health Reg Issues. 2014;4:66-74.

36. Davitkov P, Chandar AK, Hirsch A, Compan A, Silveira MG, Anthony DD, Smith S, Gideon C, Bonomo RA, Falck-Ytter Y. Treatment selection choices should not be based on benefits or costs alone: a head-to-head randomized controlled trial of antiviral drugs for hepatitis C. PLoS One. 2016;11(10):e0163945.

37. Hezode C, Fontaine H, Dorival C, Zoulim F, Larrey D, Canva V, De Ledinghen $V$, Poynard T, Samuel D, Bourliere M, et al. Effectiveness of telaprevir or boceprevir in treatment-experienced patients with HCV genotype 1 infection and cirrhosis. Gastroenterology. 2014;147(1):132-42.

38. Younossi ZM, Park H, Dieterich D, Saab S, Ahmed A, Gordon SC. The value of cure associated with treating treatment-naive chronic hepatitis $C$ genotype 1: Are the new all-oral regimens good value to society? Liver Int. 2016. doi:https://doi.org/10.1111/liv.13298. [Epub ahead of print].

39. Chhatwal J, He T, Hur C, Lopez-Olivo MA. Direct-Acting Antiviral Agents for Patients With Hepatitis C Virus Genotype 1 Infection Are Cost-Saving. Clin Gastroenterol Hepatol 2016. doi: https://doi.org/10.1016/j.cgh.2016.09.015. [Epub ahead of print].

40. Dahari H, Canini L, Graw F, Uprichard SL, Araujo ES, Penaranda G, Coquet E, Chiche L, Riso A, Renou C, et al. HCV kinetic and modeling analyses indicate similar time to cure among sofosbuvir combination regimens with daclatasvir, simeprevir or ledipasvir. J Hepatol. 2016;64(6):1232-9.

41. Peres-da-Silva A, Brandao-Mello CE, Lampe E: Prevalence of sofosbuvir resistance-associated variants in Brazilian and worldwide NS5B sequences of genotype-1 HCV. Antivir Ther. 2017. doi: https://doi.org/10.3851/IMP3131. [Epub ahead of print].

42. EASL. Recommendations on treatment of hepatitis C 2016. J Hepatol. 2017; 66(1):153-94.

43. Purchasing power parity vs market exchange rate. http://www.dawn.com/ news/1214055. Accessed on 27 Mar 2017.

\section{Submit your next manuscript to BioMed Central and we will help you at every step:}

- We accept pre-submission inquiries

- Our selector tool helps you to find the most relevant journal

- We provide round the clock customer support

- Convenient online submission

- Thorough peer review

- Inclusion in PubMed and all major indexing services

- Maximum visibility for your research

Submit your manuscript at www.biomedcentral.com/submit
Biomed Central 